\title{
Study on the Mechanism of Exercise Tolerance in Chronic Heart Failure from the Perspective of Mitochondrial Dynamics
}

\author{
Kaijing Yang and Yi Tao Xue* \\ Affiliated Hospital of Shandong University of Traditional Chinese Medicine, Jinan, China \\ *Corresponding author: Yi Tao Xue, Affiliated Hospital of Shandong University of Traditional Chinese Medicine, \\ No.16369\#, Jingshi Road, Jinan, China
}

\begin{tabular}{|c|c|}
\hline ARTICLE INFO & ABSTRACT \\
\hline Received: 戛 January 18, 2021 & $\begin{array}{l}\text { Chronic heart failure (CHF) is an important public health issue worldwide because } \\
\text { of the poor prognosis }\end{array}$ \\
\hline Published: 慧 February 02, 2021 & spot in clinical investigations. In fact, the pathophysiology of CHF is complicated and \\
\hline $\begin{array}{l}\text { itation: Kaijing Yang, Yi Tao Xue. Study } \\
\text { on the Mechanism of Exercise Tolerance in } \\
\text { Chronic Heart Failure from the Perspective }\end{array}$ & $\begin{array}{l}\text { directly affect heart function. Accordingly, the mitochondrial function will be improved or } \\
\text { restored after intervention with drugs, facilitating the rehabilitation of the patients with } \\
\text { heart failure. }\end{array}$ \\
\hline
\end{tabular}
of Mitochondrial Dynamics. Biomed J Sci \& Tech Res 33(4)-2021. BJSTR. MS.ID.005422.

\section{Introduction}

Heart failure (HF) is mainly manifested as a variety of cardiac structural or functional diseases which leads to ventricular filling and/or impaired ejection function, and cardiac output cannot meet the metabolic needs of the body's tissues, with pulmonary circulation and/or systemic circulation congestion, insufficient blood perfusion of organs and tissues. It is a serious and terminal stage of various heart diseases and is also the main cause of death, seriously threatening the health of human being. Among them, chronic heart failure (CHF) is the most important chronic cardiovascular disease in the $21^{\text {st }}$ century [1]. Due to changes in the population structure, it is expected that patients with CHF will increase in the next few years [2], and it will bring a heavy burden to the entire medical system. Therefore, it is urgent to find new treatment approaches.

Mitochondria are dynamic organelles that maintain the steady state of the entire mitochondrial network structure through continuous fusion and division [3]. This dynamic equilibrium process is called mitochondrial dynamics. The homeostasis of mitochondrial dynamics is closely related to the process of energy metabolism. In cardiomyocytes with high energy demands, maintaining the stability of mitochondrial dynamics is essential [4].
If there is a dynamic imbalance between mitochondrial fusion and fission, mitochondrial dysfunction occurs, leading to insufficient ATP production. Consequently, the insufficient ATP supply will lead to cardiomyocyte dysfunction and apoptosis, resulting in heart damage. To date an increasing evidence, indicate that abnormal energy metabolism of cardiomyocytes is an important factor which induces myocardial contractile dysfunction, leading to the development of CHF.

Studies have shown that cardiomyocytes in patients with CHF are irreversibly damaged, mitochondrial division is obvious, mitochondrial fusion protein decreases and/or mitochondrial protein increases [5], and mitochondrial function decreases and autophagy, finally resulting in heart muscle cell death. Nevertheless, mitochondrial fission only occurs in the outer mitochondrial membrane, and its regulatory proteins include Drp1, Fis1, Mff and MiD49/51. By contrast, mitochondrial fusion is regulated by the extramembrane proteins including Mfn1, Mfn2 and Opa1 proteins located in the membrane [6]. In recent years, studies have shown that the DRP1 inhibitor mdivi-1 can inhibit mitochondrial division and other ways to improve the symptoms of HF [7]. SGLT2 inhibitors can also regulate mitochondrial dynamics to protect the heart [8]. In 
addition, Elamipre tide (ELAM), a mitochondrial targeting peptide treatment, significantly increases Mfn2 and Opa1, decreases Fis1 and Drp1, and normalizes the ultrastructure of mitochondria and improves heart function [9].

A growing number of evidences show that in response to various cellular stress or damage, drug intervention can effectively help its mitochondria maintain a certain degree of balance during the cycle of fission and fusion to restore the structure of mitochondria The integrity of its functions and the cells maintain normal operation. Currently, research on mitochondrial dynamics in patients with HF are still in its infancy even some conclusions are controversial. In the future, we should proceed from the perspective of mitochondrial dynamics, conduct deeper research, and actively develop new drugs to provide new ideas and methods to treat CHF in clinical practice.

\section{References}

1. Rogers C, Bush N (2015) Heart Failure: Pathophysiology, Diagnosis Medical Treatment Guidelines, and Nursing Management. Nurs Clin North Am 50(4): 787-799.

\section{ISSN: 2574-1241}

DOI: 10.26717/BJSTR.2021.33.005422

Yi Tao Xue. Biomed J Sci \& Tech Res

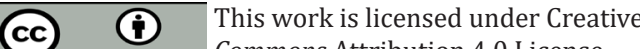

Submission Link: https://biomedres.us/submit-manuscript.php
2. Mishra S, Mohan JC, Nair T, V K Chopra, S Harikrishnan, et al. (2018) Management protocols for chronic heart failure in India. Indian Heart J $70(1): 105-127$

3. Marin-Garcia J, Akhmedov AT (2016) Mitochondrial dynamics and cell death in heart failure. Heart Fail Rev 21(2): 123-136.

4. Ferguson SM, De Camilli P (2012) Dynamin, a membrane-remodelling GTPase. Nature Reviews Molecular Cell Biology 13(2): 75-88.

5. Saotome M, Ikoma T, Hasan P, Maekawa Y (2019) Cardiac Insulin Resistance in Heart Failure: The Role of Mitochondrial Dynamics. Int J Mol Sci 20(14): 3552.

6. Qiu Z, Wei Y, Song Q, Du B, Wang H, et al. (2019) The Role of Myocardial Mitochondrial Quality Control in Heart Failure. Front Pharmacol 10: 1404

7. Givvimani S, Munjal C, Tyagi N, Sen U, Metreveli N, et al. (2012) Mitochondrial division/mitophagy inhibitor (Mdivi) ameliorates pressure overload induced heart failure. PLoS One 7(3): e32388.

8. Maejima Y (2020) SGLT2 Inhibitors Play a Salutary Role in Heart Failure via Modulation of the Mitochondrial Function. Front Cardiovasc Med 6: 186.

9. Sabbah HN, Gupta RC, Singh-Gupta V, Zhang K, Lanfear DE (2018) Abnormalities of Mitochondrial Dynamics in the Failing Heart: Normalization Following Long-Term Therapy with Elamipretide. Cardiovasc Drugs Ther 32(4): 319-328.

$\begin{array}{ll}\text { BIOMEDICAL } & \text { Assets of Publishing with us } \\ \text { RESEARCHES } & \text { - Global archiving of articles } \\ \text { - Immediate, unrestricted online access } & \text { - Rigorous Peer Review Process } \\ & \text { - Authors Retain Copyrights }\end{array}$

